\title{
ZASSENHAUS CONJECTURE FOR CYCLIC-BY-ABELIAN GROUPS
}

\author{
MAURICIO CAICEDO, LEO MARGOLIS, AND ÁNGEL DEL RÍO
}

\begin{abstract}
Zassenhaus Conjecture for torsion units states that every augmentation one torsion unit of the integral group ring of a finite group $G$ is conjugate to an element of $G$ in the units of rational group algebra $\mathbb{Q} G$. This conjecture has been proved for nilpotent groups, metacyclic groups and some other families of groups. We prove the conjecture for cyclic-by-abelian groups.
\end{abstract}

In this paper $G$ is a finite group and $R G$ denotes the group ring of $G$ with coefficients in a ring $R$. The units of $R G$ of augmentation one are usually called normalized units. In the 1960s Hans Zassenhaus established a series of conjectures about the finite subgroups of normalized units of $\mathbb{Z} G$. Namely he conjectured that every finite group of normalized units of $\mathbb{Z} G$ is conjugate to a subgroup of $G$ in the units of $\mathbb{Q} G$. These conjecture is usually denoted (ZC3), while the version of (ZC3) for the particular case of subgroups of normalized units with the same cardinality as $G$ is usually denoted (ZC2). These conjectures have important consequences. For example, a positive solution of (ZC2) implies a positive solution for the Isomorphism and Automorphism Problems (see [Seh93] for details). The most celebrated positive result for Zassenhaus Conjectures is due to Weiss [Wei91] who proved (ZC3) for nilpotent groups. However Roggenkamp and Scott founded a counterexample to the Automorphism Problem, and henceforth to (ZC2) (see Rog91 and Kli91). Later Hertweck Her01 provided a counterexample to the Isomorphism Problem.

The only conjecture of Zassenhaus that is still up is the version for cyclic subgroups namely:

Zassenhaus Conjecture for Torsion Units (ZC1). If $G$ is a finite group then every normalized torsion unit of $\mathbb{Z} G$ is conjugate in $\mathbb{Q} G$ to an element of $G$.

Besides the family of nilpotent groups, (ZC1) has been proved for some concrete groups [BH08, BHK04, HK06, LP89, LT91, Her08b, for groups having a Sylow subgroup with an abelian complement [Her06], for some families of cyclic-by-abelian groups [LB83, LT90, LS98, MRSW87, PMS84, PMRS86, dRS06, RS83, and some classes of metabelian groups not necessarily cyclic-by-abelian [MRSW87, SW86. Other results on Zassenhaus Conjectures can be found in [Seh93, Seh01] and [Seh03, Section 8]

The latest and most general result for (ZC1) on the class of cyclic-by-abelian groups is due to Hertweck Her08a who proved (ZC1) for finite groups of the form $G=A X$ with $A$ a cyclic normal subgroup of $G$ and $X$ an abelian subgroup of

2010 Mathematics Subject Classification. 16U60, 16S34; Secondary 20 C05.

Key words and phrases. Integral group ring, torsion unit, Zassenhaus Conjecture.

The research is partially supported by the Ministerio de Ciencia y Tecnología of Spain and Fundación Séneca of Murcia and Die Studienstiftung des deutschen Volkes. 
$G$. This includes the class of metacyclic groups that was not covered in previous results. The aim of this paper is to prove (ZC1) for arbitrary cyclic-by-abelian groups. Formally we prove

Theorem. Let $G$ be a finite cyclic-by-abelian group. Then every normalized torsion unit of $\mathbb{Z} G$ is conjugate in $\mathbb{Q} G$ to an element of $G$.

Our strategy uses induction on the order of the group $G$ and on the order of the torsion unit. In other words we consider a finite cyclic-by-abelian group $G$, which is a minimal counterexample to (ZC1), and $u$ a torsion unit in $\mathbb{Z} G$, which is a minimal counterexample to (ZC1). Here minimal means "of minimal order". In particular, we assume that (ZC1) holds for proper subgroups and quotients of $G$ and for units in proper subgroups of the group generated by $u$.

Most of the ideas used in this paper are either due to or inspired from the techniques introduced by Hertweck in [Her08a] which we have adapted in some steps of the proof to avoid some difficulties appearing in the general case which are not encountered in the hypothesis of Her08a. For example, the strategy in Her08a for the case when $G=A X$ with $A$ cyclic normal and $X$ abelian, consists in first proving (ZC1) for torsion units $u$ with augmentation 1 modulo $A$, with the help of a result of Cliff and Weiss for the matrix version of Zassenhaus Conjecture (Theorem 1.5) and a beautiful use of Weiss permutation module theorem Wei88, then using this to prove the results for units with augmentation 1 modulo $C_{G}(A)$ and then reducing the general case to this special case. In several steps of the proof one uses a faithful linear representation of $A$ and the fact that $C_{G}(A)=A C_{X}(A)=A Z(G)$ (and hence $C_{G}(A)$ is cyclic-by-central). In the words of Hertweck the last fact is "the main reason for assuming that $A$ is covered by an abelian subgroup-rather than assuming that $G / A$ is abelian".

In our strategy the subgroup $D=Z\left(C_{G}(A)\right)$, for $A$ a cyclic subgroup of $G$ containing $G^{\prime}$, plays a very important role. We first prove (ZC1) for units with augmentation 1 modulo $D$ using local methods over the $p$-adic integers and then we prove $(\mathrm{ZC} 1)$ for the remaining units using the so called Luthar-Passi Method. As $D$ is not cyclic-by-central it is not possible to use neither Cliff-Weiss Theorem, nor a faithful linear character of an appropriate cyclic subgroup of $G$. Instead, for the first part of the proof we adapt the $p$-adic methods of Hertweck and CliffWeiss to our situation with a careful revision of their proofs, using linear characters of $D$ with kernels not intersecting $A$, and in the second part we use a family of linear characters of $D$ with kernel not containing any normal subgroup of $G$. This introduces some difficulties in the arguments which makes the proofs more involved than in [Her08a].

\section{Notation, PRELIMINARIES AND SOME TOOLS}

In this section we establish the general notation and collect some known results, which will be used throughout the paper.

As it is costumary $\varphi$ denotes Euler's totient function. The cardinality of a set $X$ is denoted $|X|$. For every integer $n$ we let $\zeta_{n}$ denote a fixed complex primitive root of unity of order $n$. The ring of $p$-adic integers, for $p$ a prime integer, is denoted $\mathbb{Z}_{p}$

We use the standard group theoretical notation. In particular, if $G$ is a group, then $Z(G)$ denotes the center of $G, G^{\prime}$ the commutator subgroup of $G$ and $\exp (G)$ 
the exponent of $G$. If $g, h \in G$ then $|g|$ denotes the order of $g, g^{h}=h^{-1} g h$, $(g, h)=g^{-1} g^{h}=g^{-1} h^{-1} g h$ and $g^{G}$ denotes the conjugacy class of $g$ in $G$. If $X \subseteq G$ then $\langle X\rangle$ denotes the subgroup generated by $X, C_{G}(X)=\{g \in G:(x, g)=$ 1 for every $x \in X\}$, the centralizer of $X$ in $G$ and $N_{G}(X)=\left\{g \in G: X^{g} \subseteq X\right\}$, the normalizer of $X$ in $G$. Let $p$ be a prime integer. If $g$ has finite order then $g_{p}$ and $g_{p^{\prime}}$ denote the $p$-part and $p^{\prime}$-part of $g$, respectively. If $G$ has a unique $p$-Sylow subgroup (respectively a unique $p^{\prime}$-Hall subgroup) then it is denoted $G_{p}$ (respectively $G_{p^{\prime}}$ ).

In the remainder of this section $R$ stands for a commutative ring. If $N$ is a normal subgroup of $G$ then the $N$-augmentation map of $R G$ is the unique ring homomorphism $\omega_{N}: R G \rightarrow R(G / N)$ extending the natural map $G \rightarrow G / N$ and acting on $R$ as the identity. In particular $\omega=\omega_{G}$ is the augmentation map of $R G$. Let $r=\sum_{g \in G} r_{g} g \in R G$ with $r_{g} \in R$ for every $g$. For every $g \in G$, let $\varepsilon_{g}^{G}(r)$ denote the partial augmentation of $r$ in the conjugacy class of $g$ in $G$, that is

$$
\varepsilon_{g}^{G}(r)=\sum_{h \in g^{G}} r_{h} .
$$

If the group $G$ is clear from the context we simply write $\varepsilon_{g}(r)$. Conjugacy classes in $R G$ and partial augmentation are strongly related. We collect in the following remark some easy facts about this relation.

Remark 1.1. If $x \in G$ then $\varepsilon_{x}^{G}: R G \rightarrow R$ is an $R$-linear map which satisfies $\varepsilon_{x}^{G}(u v)=\varepsilon_{x}^{G}(v u)$ for every $u, v \in R G$. Using this it is easy to prove that if $u \in R G$ and $g \in G$ are conjugate in $R G$, then $\varepsilon_{g}^{G}(u)=1$ and $\varepsilon_{x}^{G}(u)=0$ for every $x \in G$ with $x \notin g^{G}$. Hence, for such $u$ and $g$ and a normal subgroup $N$ of $G$ we have $\omega_{N}(u) \neq 0$ if and only if $g \in N$ and in that case $\omega_{N}(u)=1$.

One of the main tools to study (ZC1) is the following well known result which is somehow a converse of Remark 1.1 (see e.g. [Seh93, Lemma 41.5]).

Proposition 1.2. Let $u$ be a normalized torsion unit of $\mathbb{Z} G$. Then $u$ is conjugate in $\mathbb{Q} G$ to an element of $G$ if and only if $\varepsilon_{g}^{G}(v) \geq 0$ for every $v \in\langle u\rangle$ and every $g \in G$.

Proposition 1.2 is commonly presented in the following equivalent form: A normalized torsion unit $u$ of $\mathbb{Z} G$ is conjugate in $\mathbb{Q} G$ to an element of $G$ if and only if for every $v \in\langle u\rangle$, there is $g \in G$ such that for every $x \in G$ we have $\varepsilon_{x}^{G}(v) \neq 0$ if and only if $x \in g^{G}$.

The following proposition collects some results from [Her06] and Her08a which will be very useful in our arguments.

Proposition 1.3. Let $G$ be a finite group and $p$ a prime integer.

(1) Let $R$ be a p-adic ring with quotient field $K$ and $u$ a normalized torsion unit of $R G$.

(a) Suppose $\omega_{P}(u)=1$ for $P$ a normal p-subgroup of $G$. Then $u$ is conjugate in $K G$ to an element of $P$.

(b) Suppose that the p-part of $u$ is conjugate to an element $x$ of $G$ in the units of $R G$ and $g$ is an element of $G$ such that the p-parts of $x$ and $g$ are not conjugate in $G$. Then $\varepsilon_{g}(u)=0$.

(2) Let $u$ be a torsion unit of $\mathbb{Z} G$.

(a) If $\varepsilon_{g}(u) \neq 0$ with $g \in G$ then the order of $g$ divides the order of $u$. 
(b) Assume that $\omega_{P}(u)=1$ with $P$ a cyclic normal p-subgroup of $G$. Then $u$ is conjugate in $\mathbb{Q} G$ to an element $x \in P$. If moreover, $\mathrm{C}_{G}(x)$ has a normal $p$-complement, then $u$ and $x$ are conjugate in $\mathbb{Z}_{p} G$.

We now present a matrix version of (ZC1) which was introduced in MRSW87. as a strategy to prove (ZC1) in some cases. If $k$ is a positive integer then the action of $\omega$ on the matrix entries defines a ring homomorphism $M_{k}(R G) \rightarrow M_{k}(R)$. It restricts to a group homomorphism $\mathrm{GL}_{k}(R G) \rightarrow \mathrm{GL}_{k}(R)$. Following [CW00] we let $\mathrm{SGL}_{k}(R G)$ denote the kernel of this group homomorphism. The matrix version of $(\mathrm{ZC} 1)$ is the following problem:

Problem 1.4. Let $G$ be a finite group. Is every element of finite order of $\operatorname{SGL}_{k}(\mathbb{Z} G)$ conjugate in $\mathrm{GL}_{k}(\mathbb{Q} G)$ to a diagonal matrix with diagonal entries in $G$ ?

Cliff and Weiss solved this problem for nilpotent groups and arbitrary $k$.

Theorem 1.5. CW00 If $G$ is a finite nilpotent group then Problem 1.4 has a positive solution for every $k \geq 1$ if and only if $G$ has at most one non-cyclic Sylow subgroup.

Let $N$ be a normal subgroup of $G$ with $k=[G: N]$. Then $R G$ is a $(R G, R N)$ bimodule and the right $R N$-module $R G$ is free with a basis formed by a transversal of $N$ in $G$. For every $r \in R G$ let $\rho_{N}(r)$ denote the matrix representation of left multiplication by $r$ with respect to this basis. This defines a ring homomorphism $\rho_{N}: R G \rightarrow M_{k}(R N)$. If $r$ is a unit of $R G$ such that $\omega_{N}(r)=1$ then $\rho_{N}(r) \in$ $\mathrm{SGL}_{k}(R N)$. If $x \in N$ then, by [Seh93, Lemma 41.10], we have

$$
\varepsilon_{x}^{N}\left(\operatorname{tr}\left(\rho_{N}(r)\right)\right)=\left[C_{G}(x): C_{N}(x)\right] \varepsilon_{x}^{G}(r),
$$

where $\operatorname{tr}(U)$ stands for the trace of a matrix $U \in M_{k}(R N)$. If $N$ is not commutative and $U, V \in M_{k}(R N)$ then $\operatorname{tr}(U V)$ and $\operatorname{tr}(V U)$ might be different. However, $\operatorname{tr}(U V)-\operatorname{tr}(V U) \in[R N, R N]$, where $[R N, R N]$ is the $R$ linear span of the Lie products $[a, b]=a b-b a$, with $a, b \in N$. Hence $\varepsilon_{x}^{N}(\operatorname{tr}(U V))=\varepsilon_{x}^{N}(\operatorname{tr}(V U))$ for every $x \in N$. In particular, if $U$ and $V$ are conjugate in $M_{k}(R N)$, then $\varepsilon_{x}^{N}(\operatorname{tr}(U))=\varepsilon_{x}^{N}(\operatorname{tr}(V))$.

A useful technique to deal with Zassenhaus conjecture (in the most general form) is the so called double action formalism introduced by Weiss Wei88. Let $G$ and $H$ be groups, $R$ a commutative ring and let $\alpha: H \rightarrow \mathrm{GL}_{k}(R G)$ be a group homomorphism. Then $M^{\alpha}$ denotes the right $R(H \times G)$ module $(R G)^{k}$ with multiplication defined by $x \cdot r(h, g)=\alpha(h)^{-1} x r g$ for $x \in(R G)^{k}, g \in G, h \in H$ and $r \in R$. It is easy to see that if $\beta: H \rightarrow \mathrm{GL}_{k}(R G)$ is another group homomorphism then $M^{\alpha} \cong M^{\beta}$ if and only if $\alpha$ and $\beta$ are conjugate in $\mathrm{GL}_{k}(R G)$, i.e. if and only if there exist $u \in \mathrm{GL}_{k}(R G)$ such that $\beta(h)=\alpha(h)^{u}$ for every $h \in H$. For example, if $C_{m}=\langle c\rangle$, the cyclic group of order $m$ generated by $c$, and $u$ and $v$ are torsion unit of order $m$ in $\mathrm{GL}_{k}(R G)$ then $u$ and $v$ are conjugate in $\mathrm{GL}_{k}(R G)$ if and only if the $R\left(C_{m} \times G\right)$ modules $M^{\alpha_{u}}$ and $M^{\alpha_{v}}$ are isomorphic, where $\alpha_{u}$ and $\alpha_{v}$ are the homomorphisms $C_{m} \rightarrow \mathrm{GL}_{k}(R G)$ mapping $c$ to $u$ and $v$ respectively.

Let $m$ be a positive integer, set $\Gamma=C_{m} \times G$ and let $G[m]$ denote a set of representatives of $G$-conjugacy classes of elements $g \in G$ with $g^{m}=1$. For every $g \in G$ let $[g]=\langle(c, g)\rangle \leq \Gamma$. For every prime integer $p$ and $g \in G$ let $G_{g, p}[m]=$ $\left\{h \in G[m]: g_{p}\right.$ and $h_{p}$ are conjugate in $\left.G\right\}$. For $H$ a subgroup of a group $K$ we let $\operatorname{ind}_{H}^{K} 1$ denote the character of $K$ induced from the trivial character of $H$. 
Lemma 1.6. Let $G$ be a finite nilpotent group, $u \in \mathrm{SGL}_{k}(\mathbb{Z} G)$ with $u^{m}=1$ and let $\chi$ denote the character of $M^{\alpha_{u}}$. Then

(1) For every $g \in G[m]$ we have $\chi(c, g) \in\left|C_{G}(g)\right| \mathbb{Z}$ and $\chi=\sum_{g \in G[m]} \frac{\chi(c, g)}{\left|C_{G}(g)\right|} \operatorname{ind}_{[g]}^{\Gamma} 1$.

(2) For every prime $p$ and every $g \in G[m], \sum_{h \in G_{g, p}[m]} \frac{\chi(c, h)}{\left|C_{G}(h)\right|} \operatorname{ind}_{\left[h_{p^{\prime}}\right]}^{\Gamma_{p^{\prime}}} 1$ is a proper character of $\Gamma_{p^{\prime}}$.

Proof. Cliff and Weiss [CW00, prove that $\chi=\sum_{g \in G[m]} a_{g} \operatorname{ind}_{[g]}^{\Gamma} 1$ for unique integers $a_{g}$ and $\sum_{h \in G_{g, p}[m]} a_{h} \operatorname{ind}_{\left[h_{p^{\prime}}\right]}^{\Gamma_{p^{\prime}}} 1$ is a proper character of $\Gamma_{p^{\prime}}$. So we only have to prove that $\chi(c, g)=a_{g}\left|C_{G}(g)\right|$ and this follows from

$$
\left(\operatorname{ind}_{[h]}^{\Gamma} 1\right)(c, g)= \begin{cases}\left|C_{G}(g)\right|, & \text { if } h \in g^{G} \\ 0, & \text { otherwise. }\end{cases}
$$

\section{Torsion units with $D$-Augmentation 1}

In this section $G$ is a finite group. The title of this section refers to $D=$ $Z\left(C_{G}(A)\right)$ for a cyclic subgroup $A$ of $G$ containing $G^{\prime}$ (see the introduction). So the aim of this section is to prove (ZC1) for torsion units $u$ with $\omega_{D}(u)=1$.

We start with a lemma, which seems to be folklore.

Lemma 2.1. Let $N$ be a nilpotent normal subgroup of $G$ and $u$ a torsion unit of $\mathbb{Z} G$ such that $\omega_{N}(u)=1$. Then

(1) every prime divisor of the order of $u$ divides the order of $N$ and

(2) if the order of $u$ is a power of a prime $p$, then $\omega_{N_{p}}(u)=1$.

Proof. (1) We argue by induction on the number of primes dividing $|N|$. If this number is 0 then $N=1$ and hence $u=\omega_{N}(u)=1$. Assume that $|N|$ is divisible by $p$. By hypothesis $\omega_{N / N_{p}}\left(\omega_{N_{p}}(u)\right)=\omega_{N}(u)=1$. Let $n$ be the order of $\omega_{N_{p}}(u)$. By the induction hypothesis every prime divisor of $n$ divides $\left[N: N_{p}\right]$. Moreover the order of $u^{n}$ is a power of $p$ by [Seh93, Lemma 7.5]. Let $q$ be a prime divisor of $|u|$. Then $q$ is either $p$ or a divisor of $n$. We conclude that $q$ divides $|N|$.

(2) Assume that the order of $u$ is a power of $p$ and set $u_{1}=\omega_{N_{p}}(u)$. Then $u_{1}$ is a $p$-element of $\mathbb{Z}\left(G / N_{p}\right)$ such that $\omega_{N / N_{p}}\left(u_{1}\right)=\omega_{N}(u)=1$. Since $p$ is coprime with $\left[N: N_{p}\right]$, the order of $u_{1}$ is coprime with $p$, by (1). Hence $u_{1}=1$, as desired.

The following lemma extends Her08a, Claim 5.2] where it was proved for the case where $G=A X$ with $A$ a cyclic normal subgroup of $G$ and $X$ an abelian subgroup of $G$.

Lemma 2.2. Let $A$ be a cyclic subgroup of $G$ containing $G^{\prime}$ and let $N$ be a nontrivial p-subgroup of $A$ for some prime $p$. Then $\mathrm{C}_{G}(N)$ has a normal p-complement (i.e. it has a normal $p^{\prime}$-Hall subgroup).

Proof. By replacing $G$ by $C_{G}(N)$ we may assume without loss of generality that $N$ is central in $G$. If $N_{1}$ is the unique minimal non-trivial subgroup of $N$ and $\alpha: \operatorname{Aut}\left(A_{p}\right) \rightarrow \operatorname{Aut}\left(N_{1}\right)$ is the restriction map, then the kernel of $\alpha$ is a $p$-group. Therefore the $p^{\prime}$-elements of $G$ commute with the $p$-elements of $A$. Let $H$ be a $p^{\prime}$-Hall subgroup of $G$. We will prove that $H$ is normal in $G$. Let $h \in H$ and $g \in G$. As $G / A$ is abelian, $h^{g}=a h$ for some $a \in A$. By Hall Theorem [Rob82, 9.1.7], it easily follows, that $A_{p^{\prime}} \subseteq H$. Therefore $a_{p^{\prime}} h \in H$ and in particular the order of 
$a_{p^{\prime}} h$ is coprime with $p$. Thus $\left(a_{p}, a_{p^{\prime}} h\right)=1$ and hence the order of $h^{g}$ is divisible by the order of $a_{p}$. Thus $a_{p}=1$ and we conclude that $h^{g}=a_{p^{\prime}} h \in H$.

The argument of the following lemma was already used in dRS06 and Her08a to prove that partial augmentations of elements in $G \backslash C_{G}(A)$ are non-negative in the minimal counterexamples. We need this also for elements in $G \backslash Z\left(C_{G}(A)\right)$.

Lemma 2.3. Let $A$ be a cyclic subgroup of $G$ containing $G^{\prime}$ and assume that $G / N$ satisfies (ZC1) for every non-trivial subgroup $N$ of $A$. Let $u$ be a normalized torsion unit in $\mathbb{Z} G$ and let $x \in G \backslash\left(Z\left(C_{G}(A)\right)\right)$. Then $\varepsilon_{x}(u) \geq 0$.

Proof. Let $C=C_{G}(A)$ and $D=Z(C)$. If $x \notin C$, then $N=\langle(A, x)\rangle$ is a nontrivial subgroup of $G$ contained in $A$. By hypothesis, (ZC1) holds for $G / N$. Hence $\varepsilon_{x N}^{G / N}\left(\omega_{N}(u)\right) \geq 0$, by Proposition 1.2. By [dRS06, Lemma 2], $N x^{G}=x^{G}$ and this implies that $\varepsilon_{x}^{G}(u)=\varepsilon_{x N}^{G / N}\left(\omega_{N}(u)\right) \geq 0$, as desired.

Assume that $x \in C \backslash D$. Then $(x, c) \neq 1$ for some $c \in C$. Let $N=\langle(x, c)\rangle$, a non-trivial normal subgroup of $G$. We claim that $x^{G}=N x^{G}$. Indeed, as $G^{\prime} \subseteq A$ and $(A, C)=1$, if $w, v \in C$ and $g \in G$, then $(w v, g)=(w, g)^{v}(v, g)=(w, g)(v, g)$. Thus, if $n \in N$, then $n=(x, c)^{m}=\left(x, c^{m}\right)$ for some integer $m$. If $g \in G$, then $n x^{g}=\left(x, c^{m}\right) x^{g}=\left(x, c^{m}\right) x(x, g)=x\left(x, g c^{m}\right)=x^{g c^{m}}$. This proves the claim.

We set $\bar{\alpha}=\omega_{N}(\alpha)$ for every $\alpha \in \mathbb{Z} G$. By hypothesis (ZC1) holds for $G / N$ and hence $\varepsilon_{\bar{x}}^{G / N}(\bar{u}) \geq 0$. If $u=\sum_{g \in G} u_{g} g$ with $u_{g} \in \mathbb{Z}$ for each $g$ then $\varepsilon_{x}^{G}(u)=$ $\sum_{g \in x^{G}} u_{g}=\sum_{g \in N x^{G}} u_{g}=\varepsilon_{\bar{x}}^{G / N}(\bar{u}) \geq 0$, as desired.

The following lemma comes from a closer investigation of the proof of Theorem 1.5] as given CW00.

Lemma 2.4. Let $N$ be an abelian normal subgroup of $G$ and $u$ a torsion unit in $\mathbb{Z} G$ with $\omega_{N}(u)=1$. Let $\eta$ be an irreducible character of $N$ and $n \in N$. Then

$$
\sum_{h \in \operatorname{ker} \eta}\left[C_{G}(h n): N\right] \varepsilon_{h n}^{G}(u) \geq 0
$$

Proof. Let $m$ be the order of $u$ and let $v$ be the image of $u$ under the natural permutation homomorphism $\mathbb{Z} G \rightarrow G L_{k}(\mathbb{Z} N)$. Then $v \in S G L_{k}(\mathbb{Z} N)$, where $k=$ $[G: N]$. Let $\Gamma=C_{m} \times N$, with $C_{m}=\langle c\rangle$, a cyclic group of order $m$, let $\chi$ be the character of $M^{\alpha_{v}}$ and let $N[m]=\left\{n \in N: n^{m}=1\right\}$. By [Wei91, Lemma 1] we know $\left|C_{G}(n)\right| \varepsilon_{n}^{G}(u)=\chi(c, n)$ for every $n \in N$. Moreover, $\varepsilon_{h}^{G}(u)=0$ if $h \in N \backslash N[m]$, by statement (2a) of Proposition 1.3. Combining this with Lemma 1.6 we have

$$
\begin{aligned}
\chi & =\sum_{h \in N[m]} \frac{\chi(c, h)}{\left|C_{N}(h)\right|} \operatorname{ind}_{[h]}^{\Gamma} 1=\sum_{h \in N[m]}\left[C_{G}(h): N\right] \varepsilon_{h}^{G}(u) \operatorname{ind}_{[h]}^{\Gamma} 1 \\
& =\sum_{h \in N}\left[C_{G}(h): N\right] \varepsilon_{h}^{G}(u) \operatorname{ind}_{[h]}^{\Gamma} 1
\end{aligned}
$$


and for every prime integer $p$ and every character $\psi$ of $\Gamma_{p^{\prime}}$ we have

$$
\begin{aligned}
0 & \leq\left\langle\sum_{h \in N_{n, p}[m]}\left[C_{G}(h): N\right] \varepsilon_{h}^{G}(u) \operatorname{ind}_{\left[h_{p^{\prime}}\right]}^{\Gamma_{p^{\prime}}} 1, \psi\right\rangle \\
& =\sum_{h \in N_{n, p}[m]}\left[C_{G}(h): N\right] \varepsilon_{h}^{G}(u)\left\langle\operatorname{ind}_{\left[h_{p^{\prime}}\right.}^{\Gamma_{p^{\prime}}} 1, \psi\right\rangle_{\Gamma_{p^{\prime}}} \\
& =\sum_{h \in N, h_{p}=n_{p}, \psi\left(c_{p^{\prime}}, h_{p^{\prime}}\right)=1}\left[C_{G}(h): N\right] \varepsilon_{h}^{G}(u)=\sum_{h \in N_{p^{\prime}}, \psi(1, h)=1}\left[C_{G}\left(h h^{\prime}\right): N\right] \varepsilon_{h h^{\prime}}^{G}(u),
\end{aligned}
$$

where $h^{\prime}$ is a fixed element of $N$ with $h_{p}^{\prime}=n_{p}$ and $\psi\left(c, h^{\prime}\right)=1$.

Let $\psi$ be the character of $\Gamma$ given by $\left.\psi\right|_{N}=\eta$ and $\psi(u)=\eta(n)^{-1}$. Then $\psi(c, n)=1$ and therefore applying the previous inequality for $h^{\prime}=h_{p} n$ with $h_{p} \in$ $N_{p}$ we deduce that

$$
\sum_{h \in N_{p^{\prime}} \cap \operatorname{ker} \eta}\left[C_{G}\left(h h_{p} n\right): N\right] \varepsilon_{h h_{p} n}^{G}(u) \geq 0
$$

We conclude that

$$
\sum_{h \in \text { ker } \eta}\left[C_{G}(h n): N\right] \varepsilon_{h n}^{G}(u)=\sum_{h_{p} \in N_{p} \cap \operatorname{ker} \eta} \sum_{h_{p^{\prime}} \in N_{p^{\prime}} \cap \operatorname{ker} \eta}\left[C_{G}\left(h_{p^{\prime}} h_{p} n\right): N\right] \varepsilon_{h_{p^{\prime}} h_{p} n}^{G}(u) \geq 0
$$

as desired.

The following theorem is an adjustment of [Her08a, Theorem 5.1] to our situation.

Theorem 2.5. Let $G$ be a finite group and $A$ a cyclic normal subgroup of $G$ containing $G^{\prime}$. Set $D=Z\left(C_{G}(A)\right)$ and let $u$ be a torsion unit of $\mathbb{Z} G$ with $\omega_{D}(u)=1$. If the order of $u$ is a power of a prime $p$, then $u$ is conjugate in $\mathbb{Z}_{p} G$ to an element of $D_{p}$.

Proof. By statement (1a) of Proposition 1.3, $u$ is conjugate in $\mathbb{Q} G$ to an $x \in D_{p}$. Let $R$ be a $p$-adic ring with quotient field $K$ containing a root of unity of order the exponent of $G$. We will proof that $u$ is conjugate to $x$ in $R G$. Then by CR62, $30.25]$ the conjugation already takes place in $\mathbb{Z}_{p} G$.

Set $L=C_{G}\left(D_{p}\right), E=C_{G}(x)$ and let $Q$ be the normal $p$-complement of $L$, that exists by Lemma 2.2. Note that $L$ and $E$ are normal in $G$, for they contain $A$, and $Q$ is also normal in $G$ for it is a characteristic subgroup of $L$.

The primitive central idempotents of $K Q$ belong to $R Q$, because the order of $Q$ is invertible in $R$. Moreover $G$ acts on these primitive central idempotents by conjugation. Let $\epsilon_{1}, \ldots, \epsilon_{\beta}$ be the sums of the $G$-orbits of this action. Then $R G=$ $\prod_{i=1}^{\beta} \epsilon_{i} R G$ and therefore it is enough to show that $\epsilon_{i} u$ is conjugate to $\epsilon_{i} x$ in $\epsilon_{i} R G$ for every $i$. Note that $\epsilon_{i} u$ is conjugate to $\epsilon_{i} x$ in $\epsilon_{i} K G$ and a primitive idempotent of $K Q$ stays primitive in $K L$ by Greens Indecomposability Theorem, since $L / Q$ is a $p$-group CR62, 19.23].

So fix one primitive central idempotent $f$ of $K Q$ and let $\epsilon$ be the sum of the $G$-conjugates of $f$. We have to prove that $\epsilon u$ and $\epsilon x$ are conjugate in $\epsilon R G$. Let $e$ be the sum of different $L$-conjugates of $f$ and write $e=e_{1}+\ldots+e_{m}$ with 
orthogonal primitive idempotents of $R Q$. Let $T=C_{G}(e)$ and let $\left\{1=s_{1}, \ldots, s_{n}\right\}$ be a transversal of $G / T$. Then

$$
\epsilon=\sum_{i=1}^{m} \sum_{j=1}^{n} e_{i}^{s_{j}^{-1}}
$$

is a decomposition into primitive orthogonal idempotents of $\epsilon R L$. So by [Her08a, Lemma 4.6] there exist $g_{i j} \in G$ such that

$$
v=\sum_{i=1}^{m} \sum_{j=1}^{n} e_{i}^{s_{j}^{-1}} x^{g_{i j}}
$$

is conjugate to $\epsilon u$ in $R G$. In particular $|v|=|\epsilon u|=|\epsilon x|$. Let $C=\langle c\rangle$ a cyclic group with the same order as $v$ and consider the $R(C \times G)$-modules $M=M^{\alpha}$ and $N=M^{\beta}$, with $\alpha=\alpha_{v}$ and $\beta=\alpha_{\epsilon x}$. (We are abusing the notation by writing $\alpha_{v}$ and $\alpha_{\epsilon x}$ instead of $\alpha_{v+(1-\epsilon)}$ and $\alpha_{\epsilon x+(1-\epsilon)}$, respectively.) We have to show that $v$ is also conjugate to $\epsilon x$ in $\epsilon R G$ or equivalently that $M$ and $N$ are isomorphic as $R(C \times G)$-modules.

As $x \in D_{p}$ and $e_{i} \in R L$ and both $D_{p}$ and $L$ are normal in $G$, every $G$-conjugate of $x$ belongs to $D_{p}$ and every $G$-conjugate of $e_{i}$ belong to $R L$. On the other hand $\left(D_{p}, L\right)=1$ and therefore every $G$-conjugate of $x$ commutes with every $G$-conjugate of $e_{i}$. Using this it easily follows that each $R G e_{i}^{s_{j}^{-1}}$ is a submodule of both $M$ and $N$. We set $M_{i j}=M e_{i}^{s_{j}^{-1}}$ and $N_{i j}=N e_{i}^{s_{j}^{-1}}$, i.e. both $M_{i j}$ and $N_{i j}$ are $R G e_{i}^{s_{j}^{-1}}$, considered as submodules of $M$ and $N$ respectively. The strategy of the proof consists in pairing isomorphic $M_{i j}$ 's and $N_{i j}$ 's.

Firstly observe that if $g \in G$, then every two primitive idempotents $\varepsilon_{1}$ and $\varepsilon_{2}$ of $R Q e^{g}$ are conjugate in $R L e^{g}$, since $K L e^{g}$ is simple and hence $R L \varepsilon_{1} \cong R L \varepsilon_{2}$ (see e.g. Theorem 6.7, Proposition 16.16 and Problem 6.14 in CR62). If $e_{i}^{s_{j}^{-1}}=e_{1}^{w}$ with $w$ a unit in RQe then $a \mapsto a w$ is an isomorphism $N_{1 j} \rightarrow N_{i j}$. Secondly, if $q \in E$, then the map $a \mapsto a q$ is an isomorphism $N e_{i} \rightarrow N e_{i}^{q}$. Therefore, if we choose the transversal $\left\{s_{j}: 1 \leq j \leq n\right\}=\left\{h_{j_{2}} q_{j_{1}} \mid 1 \leq j_{1} \leq n_{1}, 1 \leq j_{2} \leq n_{2}\right\}$, with $\left\{h_{1}, \ldots, h_{n_{2}}\right\}$ a transversal of $G / T E$ and $\left\{q_{1}, \ldots, q_{n_{1}}\right\}$ a transversal of $E / E \cap T$ (which is also a transversal of $T E / T$ ), and denote $N_{i j}=N_{i\left(j_{1}, j_{2}\right)}$ if $s_{j}=h_{j_{2}} q_{j_{1}}$. Then we have

$$
N=\bigoplus_{i, j} N_{i j} \cong \bigoplus_{j_{2}=1}^{n_{2}}\left(N_{1\left(1, j_{2}\right)}\right)^{m n_{1}}
$$

If we have $g_{i j} s_{j} \equiv s_{j_{0}} \bmod T$ then $N_{i j_{0}} \cong M_{i j}$ via $a \rightarrow a g_{i j}$. So if $g_{i j} s_{j} \equiv h_{j_{2}}$ $\bmod T E$ we can pick a suitable $q_{j_{1}}$ such that by setting $s_{j_{0}}=h_{j_{2}} q_{j_{1}}$ we have $g_{i j} s_{j} \equiv s_{j_{0}}$ mod $T$ and this gives $M_{i j} \cong N_{1,\left(1, j_{2}\right)}$.

Set $X_{j_{2}}=\left\{(i, j) \mid g_{i j} s_{j} \equiv h_{j_{2}} \bmod T E\right\}$. By the previous paragraph, the isomorphism $M \cong N$ will follow from (2.2) provided $\left|X_{j_{2}}\right|=m n_{1}$. The remainder of the proof is dedicated to prove this equality. For this we will use a representation of $\epsilon K G$ and investigate the multiplicities of eigenvalues of $\epsilon x$ and $v$ under this representation. They are the same for $\epsilon x$ and $v$ are conjugate in $\epsilon K G$. This is also the strategy in the proof of [Her08a, Theorem 5.1]. However the representation used by Hertweck was constructed using a faithful linear representation of $A_{p}$ and here we need to use a linear representation of $D_{p}$. This representation cannot be faithful if $D_{p}$ is not cyclic. Instead we use a subgroup $H$ of $G$ such that $G / H$ is 
cyclic and $H \cap A=1$ and then consider a linear representation of $D_{p}$ with kernel $H$. The existence of such $H$ follows easily by observing that if $H$ is a maximal subgroup of $D_{p}$ not intersecting $A$, then $D_{p} / H$ is cyclic because otherwise $D_{p} / H$ contains a direct product $\langle c\rangle \times\langle d\rangle$ of cyclic groups of order $p$. This would yield to a contradiction using the maximality of $H$ and the fact that $A$ is cyclic.

Let $\pi$ be the linear character of a representation of $D_{p}$ with kernel $H$ and let $\psi$ be the sum of all irreducible characters of $Q$, that do not vanish on $e$. So $\psi(f)=1$ for every primitive idempotent $f$ of $R Q$ satisfying ef $\neq 0$ and hence $\psi(1)=m$. Let $\rho$ be a representation of $Q \times D_{p}$ affording $\psi \otimes \pi$. This can be chosen satisfying $\rho\left(e_{i}\right)=E_{i}$, where $E_{i}$ denotes the elementary matrix with 1 in the $i$-th diagonal entry and 0 anywhere else. Then $\rho\left(e_{i} y\right)=\pi(y) E_{i}$ for any $i$ and $y \in D_{p}$. Let $\chi=\operatorname{ind}_{Q \times D_{p}}^{T}(\psi \otimes \pi)$, let $\Delta$ be a representation of $T$ affording $\chi$ and let $\left\{t_{1}, \ldots, t_{k}\right\}$ be a transversal of $T / Q \times D_{p}$. Then, after a suitable conjugation one may assume that

$$
\Delta\left(\sum_{i=1}^{m} e_{i} y_{i}\right)=\operatorname{diag}\left(\pi\left(y_{i}^{t_{j}}\right) \mid 1 \leq i \leq m, 1 \leq j \leq k\right) \in M_{m k}(K)
$$

for every $y_{1}, \ldots, y_{m} \in D_{p}$ Denote by $\bar{\Delta}: \mathrm{M}_{n}(e K T) \rightarrow \mathrm{M}_{n m k}(K)$ the map which acts like $\Delta$ componentwise.

As right $K G$-modules we have $\epsilon K G=\sum_{j=1}^{n} e^{s_{j}^{-1}} K G \cong(e K G)^{n}$ and so

$$
\epsilon K G \cong \operatorname{End}_{K G}(\epsilon K G) \cong \mathrm{M}_{n}\left(\operatorname{End}_{K G}(e K G)\right) \cong \mathrm{M}_{n}(e K G e)
$$

Moreover, $e K G e=e \sum_{j=1}^{n} s_{j} K T e=e \sum_{j=1}^{n} e^{s_{j}^{-1}} s_{j}^{-1} K T=e K T$, since $e$ is orthogonal to any different conjugate of itself. So (2.3) gives an isomorphism $\delta: \epsilon K G \cong$ $\mathrm{M}_{n}(e K T)$, which satisfies $\delta\left(\sum_{j=1}^{n} e^{s_{j}^{-1}} y_{j}\right)=\operatorname{diag}\left(e y_{1}^{s_{1}}, \ldots, e y_{n}^{s_{n}}\right)$ for $y_{1}, \ldots, y_{k} \in K T$.

Set $\hat{\Delta}=\bar{\Delta} \circ \delta$. Then we have

$\hat{\Delta}(\epsilon x)=\bar{\Delta}\left(\operatorname{diag}\left(e x^{s_{1}}, \ldots, e x^{s_{n}}\right)\right)=\operatorname{diag}\left(\pi\left(x^{s_{j} t_{l}}\right): 1 \leq i \leq m, 1 \leq j \leq n, 1 \leq l \leq k\right)$.

Observe that the index $i$ only affects the diagonal entries of $\hat{\Delta}(\epsilon x)$ by repeating each entry $m$ times. Furthermore $\left\{s_{j} t_{l}: 1 \leq j \leq n, 1 \leq l \leq k\right\}$ is a transversal of $G /\left(Q \times D_{p}\right)$. Note that if $x^{g} \neq x$, then $x^{g}=a x$ with $1 \neq a \in A$ and so $\pi\left(x^{g}\right)=\pi(a) \pi(x) \neq \pi(x)$ by the choice of $\pi$. So, the diagonal of $\hat{\Delta}(\epsilon x)$ is formed by $[G: E]$ different entries each with multiplicity $m\left[E: Q \times D_{p}\right]$.

On the other hand we have

$$
\begin{aligned}
\hat{\Delta}(v) & =\hat{\Delta}\left(\sum_{i=1}^{m} \sum_{j=1}^{n} e_{i}^{s_{j}^{-1}} x^{g_{i j}}\right)=\bar{\Delta}\left(\operatorname{diag}\left(\sum_{i=1}^{m} e_{i} x^{g_{i 1} s_{1}}, \ldots, \sum_{i=1}^{m} e_{i} x^{g_{i n} s_{n}}\right)\right) \\
& =\operatorname{diag}\left(\pi\left(x^{g_{i j} s_{j} t_{l}}\right) \mid 1 \leq i \leq m, 1 \leq j \leq n, 1 \leq l \leq k\right)
\end{aligned}
$$

Let $\left\{v_{1}, \ldots, v_{r}\right\}$ be a transversal of $T / T \cap E$ and set $Y_{j_{2}}=\left\{(i, j, l): g_{i j} s_{j} t_{l} \equiv h_{j_{2}}\right.$ $\bmod E\}$ and $\bar{Y}_{j_{2}}=\left\{(i, j, l): g_{i j} s_{j} v_{l} \equiv h_{j_{2}} \bmod E\right\}$. Arguing as in the previous paragraph we deduce that the multiplicity of $\pi\left(x^{h_{j_{2}}}\right)$ in $\hat{\Delta}(v)$ is $\left|Y_{j_{2}}\right|=[T \cap E$ : $\left.Q \times D_{p}\right]\left|\bar{Y}_{j_{2}}\right|$. As $v$ and $\epsilon x$ are conjugate in $\epsilon K G$ we deduce that $m\left[E: Q \times D_{p}\right]=$ $\left[T \cap E: Q \times D_{p}\right]\left|\bar{Y}_{j_{2}}\right|$ and therefore $\left|\bar{Y}_{j_{2}}\right|=m\left[E: Q \times D_{p}\right] /\left[T \cap E: Q \times D_{p}\right]=m[E:$ 
$T \cap E]=m n_{1}$. Then $(i, j, l) \mapsto(i, j)$ defines a bijective map $\bar{Y}_{j_{2}} \rightarrow X_{j_{2}}$. Therefore $\left|X_{j_{2}}\right|=m n_{1}$ as desired.

Corollary 2.6. Let $G$ be a finite group and $A$ a normal cyclic subgroup of $G$ containing $G^{\prime}$. Let $D=Z\left(C_{G}(A)\right)$ and $u$ a torsion unit in $\mathbb{Z} G$ satisfying $\omega_{D}(u)=1$. Then $u$ is conjugate in $\mathbb{Q} G$ to an element of $D$.

Proof. Since $G / A$ is abelian there exists some $b \in G$ such that $\omega_{A}(u)=b A$. We claim that $\left\{n \in D: \varepsilon_{n}(u) \neq 0\right\}$ is contained in $b A$. Indeed, let $p$ be some prime dividing $|u|$. By Theorem [2.5] $u_{p}$ is conjugate in $\mathbb{Z}_{p} D$ to some $n_{p, 0} \in D$. By statement (1b) of Proposition 1.3, if $\varepsilon_{n}(u) \neq 0$ then $n_{p}$ is conjugate to $n_{p, 0}$, so $n_{p} A=n_{p, 0} A$. On the other hand we have that $b_{p} A=\omega_{A}\left(u_{p}\right)$ is conjugate to $n_{p, 0} A=\omega_{A}\left(n_{p, 0}\right)$, so $b_{p} A=n_{p, 0} A$ and hence $b_{p} A=n_{p} A$ for all primes $p$ dividing $|u|$ and all $n \in N$ with $\varepsilon_{n}(u) \neq 0$. As $|n|$ divides $|u|$, by statement (2a), we deduce that $n A=b A$ and the claim is proved.

Assume that the statement of the corollary is false. Then, by Proposition 1.1 and Lemma 2.3. there exists an $n \in G$ such that $\varepsilon_{n}(u)<0$. So $n \in b A$, by the previous paragraph. As in the proof of Theorem 2.5 there exists a subgroup $H$ in $D$ such that $H \cap A=1$ and $D / H$ is cyclic. So $D$ has a linear character $\eta$ with kernel $H$. Then, by Lemma 2.4, we have $0 \leq \sum_{h \in H}\left[C_{G}(h n): D\right] \varepsilon_{h n}(u)$. But if $\varepsilon_{h n}(u) \neq 0$ with $h \in H_{p^{\prime}}$ then $h n \in b A \cap n H=n A \cap n H=n(A \cap H)=\{n\}$. Hence

$$
0 \leq \sum_{h \in H}\left[C_{G}(h n): D\right] \varepsilon_{h n}(u)=\left[C_{G}(n): D\right] \varepsilon_{n}(u)<0,
$$

a contradiction.

\section{Reduction to torsion units of $D$-Augmentation 1}

In this section we pursue another idea from Her08a. In order to reduce the proof of (ZC1) to the case of units of $A$-augmentation 1, with $A$ a cyclic normal subgroup of $G$, Hertweck studied the multiplicities of the image of a representation of $G$ induced from a faithful linear character of $A$. In our study we need to replace $A$ by $D=Z\left(C_{G}(A)\right)$. Now $D$ may not be cyclic and in that case it does not have any faithful linear character. Alternatively we consider linear characters of $D$, whose kernel does not contain any non-trivial normal subgroup of $G$. Observe that if $D$ is cyclic then the characters satisfying this condition are precisely the faithful linear characters of $D$.

Let $N$ be an abelian normal subgroup of $G$. Then for every linear character $\psi$ of $N$ and every $u \in \mathbb{C} G$ one has

$$
\psi^{G}(u)=\sum_{n \in N} \psi(n)\left[C_{G}(n): N\right] \varepsilon_{n}^{G}(u),
$$

where $\psi^{G}$ represents the character induced from $\psi$. This can be checked directly by observing that both sides of the equality define linear maps on $\mathbb{C} G$ and checking the formula for the elements of $G$. It can be also proved using [Seh93, Lemma 41.10]. If $N=\left\{n_{1}, \ldots, n_{m}\right\}$ and $\psi_{1}, \ldots, \psi_{m}$ are the linear characters of $N$ then (3.4) yields the following

$$
\left(\begin{array}{c}
\psi_{1}^{G}(u) \\
\vdots \\
\psi_{m}^{G}(u)
\end{array}\right)=T\left(\begin{array}{c}
{\left[C_{G}\left(n_{1}\right): N\right] \varepsilon_{n_{1}}^{G}(u)} \\
\vdots \\
{\left[C_{G}\left(n_{m}\right): N\right] \varepsilon_{n_{m}}^{G}(u)}
\end{array}\right),
$$


where $T=\left(\psi_{i}\left(n_{j}\right)\right)$, the character table of $N$. By the Orthogonality Relations the transpose conjugate of $T$ is $m T^{-1}$. Multiplying by this matrix we obtain

$$
\left|C_{G}(x)\right| \varepsilon_{x}^{G}(u)=\sum_{i=1}^{m} \overline{\psi_{i}(x)} \psi_{i}^{G}(u) \quad \text { for every } x \in N \text { and } u \in \mathbb{C} G .
$$

Let

$$
\begin{aligned}
\mathbb{K}=\mathbb{K}_{N}= & \{K \leq N: N / K \text { is cyclic and } \\
& K \text { does not contain any non-trivial normal subgroup of } G\} .
\end{aligned}
$$

For every $K \in \mathbb{K}$ we select a linear character $\psi_{K}$ of $K$ with kernel $K$ and let $\rho_{K}$ be a representation of $G$ affording the induced character $\psi_{K}^{G}$. Observe that if $K_{1}$ and $K_{2}$ are conjugate in $G$ then $\psi_{K_{1}}^{G}=\psi_{K_{2}}^{G}$ and therefore we may assume that $\rho_{K_{1}}=\rho_{K_{2}}$. Let $\mathcal{C}_{\mathbb{K}}$ be the set of conjugacy classes in $G$ of elements of $\mathbb{K}$. For every $C \in \mathcal{C}_{\mathbb{K}}$ select a representative $K_{C}$ of $C$. Let $\mathbb{Q}_{K}=\mathbb{Q}\left(\zeta_{[N: K]}\right)$.

For a square matrix $U$ with entries in $\mathbb{C}$ and $\alpha \in \mathbb{C}$ let $\mu_{U}(\alpha)$ denote the multiplicity of $\alpha$ as eigenvalue of $U$. If $U^{m}=I$ and $\alpha$ is a root of unity then we have the following formula (see [LP89])

$$
\mu_{U}(\alpha)=\frac{1}{m} \sum_{d \mid m} \operatorname{tr}_{\mathbb{Q}\left(\zeta_{m}^{d}\right) / \mathbb{Q}}\left(\operatorname{tr}\left(U^{d}\right) \alpha^{-d}\right) .
$$

This formula is the bulk of the Luthar-Passi Method.

Lemma 3.1. Let $G$ be a finite group such that (ZC1) holds for every proper quotient of $G$. Let $N$ be an abelian normal subgroup of $G$. Let $u$ be a unit of $\mathbb{Z} G$ with $\omega_{N}(u) \neq 1$ and let $x \in N$.

(1) Then

$$
\left|C_{G}(x)\right| \varepsilon_{x}(u)=\sum_{K \in \mathbb{K}} \operatorname{tr}_{\mathbb{Q}_{K} / \mathbb{Q}}\left(\overline{\psi_{K}(x)} \psi_{K}^{G}(u)\right) .
$$

(2) Assume moreover that $m=|u|, f=\left|\omega_{N}(u)\right|, x^{m}=1$ and $u^{d}$ is conjugate in $\mathbb{Q} G$ to an element of $G$ for every $1 \neq d \mid m$. Then for every $h \mid f$ with $h \neq 1$ we have

$$
\begin{aligned}
\sum_{K \in \mathbb{K}}\left[\mathbb{Q}_{K}: \mathbb{Q}\right] \mu_{\rho_{K}(u)}\left(\psi_{K}(x)\right)= & \frac{\varphi(m)}{m}\left|C_{G}(x)\right| \varepsilon_{x}(u)+ \\
& \frac{1}{h} \sum_{K \in \mathbb{K}}\left[\mathbb{Q}_{K}: \mathbb{Q}\right] \mu_{\rho_{K}\left(u^{h}\right)}\left(\psi_{K}\left(x^{h}\right)\right)
\end{aligned}
$$

(3) Assume moreover that $G^{\prime}$ is cyclic and $u^{f}$ is conjugate in $\mathbb{Q} G$ to an element $y$ of $N$. Let $u_{C}=\left|\left\{g \in G: x^{f} \in y^{G} K_{C}^{g}\right\}\right|$ for $C \in \mathcal{C}_{\mathbb{K}}$. Then we have

$$
\sum_{K \in C} \mu_{\rho_{K}\left(u^{f}\right)}\left(\psi_{K}\left(x^{f}\right)\right)=\frac{\left[C_{G}(y): N\right]}{\left|N_{G}\left(K_{C}\right)\right|} u_{C} .
$$

Proof. (1) Let $\psi$ be a linear character of $N$ such that the kernel of $\psi$ contains a non-trivial normal subgroup $U$ of $G$. Then $\psi=\phi \circ \omega_{U}$ for a linear character $\phi$ of $G / U$. By the induction hypothesis $\omega_{U}(u)$ is conjugate in $\mathbb{Q}(G / U)$ to an element of $G / U$. Moreover $\omega_{N / U}\left(\omega_{U}(u)\right)=\omega_{N}(u) \neq 1$ and therefore $\varepsilon_{n U}\left(\omega_{U}(u)\right)=0$ for every $n \in N$. Then (3.4) yields $\psi^{G}(u)=\phi^{G}\left(\omega_{U}(u)\right)=0$. Hence we can drop in (3.5) all the summands labeled by linear characters of $N$ whose kernel is not in $\mathbb{K}$. The 
remaining characters are those of the form $\sigma \circ \psi_{K}$ for a $K \in \mathbb{K}$ and $\sigma \in \operatorname{Gal}\left(\mathbb{Q}_{K} / \mathbb{Q}\right)$. Hence

$\left|C_{G}(x)\right| \varepsilon_{x}(u)=\sum_{K \in \mathbb{K}} \sum_{\sigma \in \operatorname{Gal}\left(\mathbb{Q}_{K}: \mathbb{Q}\right)} \overline{\sigma \circ \psi_{K}(x)}\left(\sigma \circ \psi_{K}^{G}\right)(u)=\sum_{K \in \mathbb{K}} \operatorname{tr}_{\mathbb{Q}_{K} / \mathbb{Q}}\left(\overline{\psi_{K}(x)} \psi_{K}^{G}(u)\right)$,

as desired.

(2) Let $d$ be a divisor of $m$ such that $d \neq 1$ and $\omega_{N}\left(u^{d}\right) \neq 1$. By hypothesis $u^{d}$ is conjugate to an element of $G$ which does not belong to $N$, by Remark 1.2. Therefore $\varepsilon_{n}\left(u^{d}\right)=0$ for every $n \in N$. Hence, by (3.7) we have $\sum_{K \in \mathbb{K}} \operatorname{tr}_{\mathbb{Q}_{K} / \mathbb{Q}}\left(\left(\psi_{K}^{G}\right)\left(u^{d}\right) \psi_{K}(n)^{-1}\right)=0$. Thus,

$$
\text { if } f \nmid d \mid m, d \neq 1 \text { and } n \in N \text { then } \sum_{K \in \mathbb{K}} \operatorname{tr}_{\mathbb{Q}_{K} / \mathbb{Q}}\left(\left(\psi_{K}^{G}\right)\left(u^{d}\right) \psi_{K}(n)^{-1}\right)=0 .
$$

For every $K \in \mathbb{K}$ and every integer $d$ we use the notation $\mu(K, d)=\mu_{\rho_{K}\left(u^{d}\right)}\left(\psi_{K}\left(x^{d}\right)\right)$, the multiplicity of $\psi_{K}\left(x^{d}\right)$ as an eigenvalue of $\rho_{K}\left(u^{d}\right)$. By (3.6), for every $e \mid m$ we have

$$
\begin{aligned}
& \sum_{K \in \mathbb{K}}\left[\mathbb{Q}_{K}: \mathbb{Q}\right] \mu(K, e)= \\
& \frac{e}{m} \sum_{K \in \mathbb{K}} \sum_{d \mid(m / e)}\left[\mathbb{Q}_{K}: \mathbb{Q}\right] \operatorname{tr}_{\mathbb{Q}\left(\zeta_{m}^{e d}\right) / \mathbb{Q}}\left(\psi_{K}^{G}\left(u^{e d}\right) \psi_{K}(x)^{-e d}\right) .
\end{aligned}
$$

Let $d \mid m$ and $\alpha=\psi_{K}^{G}\left(u^{d}\right) \psi_{K}(x)^{-d}$. Clearly the image of $\psi_{K}$ is $\mathbb{Q}_{K}$ and $\psi_{K}^{G}\left(u^{d}\right) \in$ $\mathbb{Q}\left(\zeta_{m}^{d}\right)$. Moreover, $x^{m}=1$, by hypothesis. Thus $\alpha \in \mathbb{Q}_{K} \cap \mathbb{Q}\left(\zeta_{m}^{d}\right)$. Let $L=\mathbb{Q}_{K}\left(\zeta_{m}^{d}\right)$. Then $\left[L: \mathbb{Q}_{K}\right] \operatorname{tr}_{\mathbb{Q}_{K} / \mathbb{Q}}(\alpha)=\left(\operatorname{tr}_{\mathbb{Q}_{K} / \mathbb{Q}} \circ \operatorname{tr}_{L / \mathbb{Q}_{K}}\right)(\alpha)=\operatorname{tr}_{L / \mathbb{Q}}(\alpha)=\left(\operatorname{tr}_{\mathbb{Q}\left(\zeta_{m}^{d}\right) / \mathbb{Q}} \circ\right.$ $\left.\operatorname{tr}_{L / \mathbb{Q}\left(\zeta_{m}^{d}\right)}\right)(\alpha)=\left[L: \mathbb{Q}\left(\zeta_{m}^{d}\right)\right] \operatorname{tr}_{\mathbb{Q}\left(\zeta_{m}^{d}\right) / \mathbb{Q}}(\alpha)$. Therefore $\left[\mathbb{Q}\left(\zeta_{m}^{d}\right): \mathbb{Q}\right] \operatorname{tr}_{\mathbb{Q}_{K} / \mathbb{Q}}(\alpha)=\left[\mathbb{Q}_{K}:\right.$ $\mathbb{Q}] \operatorname{tr}_{\mathbb{Q}\left(\zeta_{m}^{d}\right) / \mathbb{Q}}(\alpha)$. This equality together with (3.7) yields

$$
\begin{aligned}
& \sum_{K \in \mathbb{K}}\left[\mathbb{Q}_{K}: \mathbb{Q}\right] \operatorname{tr}_{\mathbb{Q}\left(\zeta_{m}^{d}\right) / \mathbb{Q}}\left(\psi_{K}^{G}\left(u^{d}\right) \psi_{K}(x)^{-d}\right)= \\
& {\left[\mathbb{Q}\left(\zeta_{m}^{d}\right): \mathbb{Q}\right] \sum_{K \in \mathbb{K}} \operatorname{tr}_{\mathbb{Q}_{K} / \mathbb{Q}}\left(\psi_{K}^{G}\left(u^{d}\right) \psi_{K}(x)^{-d}\right)=} \\
& {\left[\mathbb{Q}\left(\zeta_{m}^{d}\right): \mathbb{Q}\right]\left|C_{G}\left(x^{d}\right)\right| \varepsilon_{x^{d}}\left(u^{d}\right) .}
\end{aligned}
$$

Moreover, by (3.10), this is 0 provided $f \nmid d \mid m$ and $d \neq 1$. Thus, for $e=1$ and $1 \neq h \mid f$, 3.11) can be reduced to the following

$$
\begin{aligned}
& \sum_{K \in \mathbb{K}}\left[\mathbb{Q}_{K}: \mathbb{Q}\right] \mu(K, 1)= \\
& \frac{\left[\mathbb{Q}\left(\zeta_{m}\right): \mathbb{Q}\right]\left|C_{G}(x)\right|}{m} \varepsilon_{x}(u)+\frac{1}{m} \sum_{K \in \mathbb{K}} \sum_{h|d| m}\left[\mathbb{Q}_{K}: \mathbb{Q}\right] \operatorname{tr}_{\mathbb{Q}\left(\zeta_{m}^{d}\right) / \mathbb{Q}}\left(\psi_{K}^{G}\left(u^{d}\right) \psi_{K}(x)^{-d}\right)= \\
& \frac{\varphi(m)\left|C_{G}(x)\right|}{m} \varepsilon_{x}(u)+\frac{1}{h} \frac{h}{m} \sum_{K \in \mathbb{K}} \sum_{d \mid(m / h)}\left[\mathbb{Q}_{K}: \mathbb{Q}\right] \operatorname{tr}_{\mathbb{Q}\left(\zeta_{m}^{h d}\right) / \mathbb{Q}}\left(\psi_{K}^{G}\left(u^{h d}\right) \psi_{K}(x)^{-h d}\right)= \\
& \frac{\varphi(m)\left|C_{G}(x)\right|}{m} \varepsilon_{x}(u)+\frac{1}{h} \sum_{K \in \mathbb{K}}\left[\mathbb{Q}_{K}: \mathbb{Q}\right] \mu(K, h),
\end{aligned}
$$

where in the last equality we have used (3.11) for $e=h$. This proves (3.8).

(3) Finally assume that $G^{\prime}$ is cyclic and $u^{f}$ is conjugate in $\mathbb{Q} G$ to $y \in N$. Then $\rho_{K}\left(u^{f}\right), \rho_{K}(y)$ and $\operatorname{diag}\left(\psi_{K}\left(y^{g}\right): g \in T\right)$ are conjugate in the matrices over $\mathbb{C}$, 
where $T$ is a transversal of $G / N$. Observe that $\psi_{K}\left(y^{g}\right)=\psi_{K}\left(y^{h}\right)$ if and only if $\psi_{K}((y, g))=\psi_{K}((y, h))$ if and only if $(y, g)(y, h)^{-1} \in K$ if and only if $(y, g)=(y, h)$ (because $K \cap G^{\prime}=1$ ), if and only if $g h^{-1} \in C_{G}(y)$. Therefore each eigenvalue of $\rho_{K}\left(u^{f}\right)$ has multiplicity $\left[C_{G}(y): N\right]$. On the other hand $\psi_{K}\left(x^{f}\right)$ is an eigenvalue of $\rho_{K}\left(u^{f}\right)$ if and only if $\psi_{K}\left(x^{f}\right)=\psi_{K}\left(y^{g}\right)$ for some $g \in G$, if and only if $x^{f} \in y^{G} K$. Therefore if $C \in \mathcal{C}_{\mathbb{K}}$ and $K_{C}$ is a representative of $C$ then

$$
\sum_{K \in C} \mu_{\rho_{K}\left(u^{f}\right)}\left(\psi_{K}\left(x^{f}\right)\right)=\frac{1}{\left|N_{G}\left(K_{C}\right)\right|} \sum_{g \in G, x^{f} \in y^{G} K_{C}^{g}}\left[C_{G}(y): N\right]=\frac{\left[C_{G}(y): N\right]}{\left|N_{G}\left(K_{C}\right)\right|} u_{C},
$$

as desired.

Remark 3.2. Let $A$ be a cyclic normal subgroup of $G$ containing $G^{\prime}$. Clearly every element of $\mathbb{K}$ does not intersect $A$ and $Z(G)$. Conversely, let $H$ be a subgroup of $G$ containing a non-trivial normal subgroup $U$ of $G$ and such that $H \cap Z(G)=1$. If $1 \neq n \in U$ then $1 \neq(n, g) \in A \cap U$ for some $g \in G$ and therefore $A \cap H \neq 1$. Thus, for every abelian subgroup $N$ of $G$ we have $\mathbb{K}_{N}=\{K \leq N: A \cap K=Z(G) \cap K=$ 1 and $N / K$ is cyclic $\}$.

Observe that $\mathbb{K}_{N}$ can be empty. For example, this is the case if $N \cap Z(G)$ is not cyclic.

Lemma 3.3. Assume that $A$ is a cyclic subgroup of $G$ containing $G^{\prime}$. Let $N$ be a an abelian subgroup of $G$ containing $A$ and $\mathbb{K}=\mathbb{K}_{N}$. Then for every $K \in \mathbb{K}$ we have $|\mathbb{K}| \leq|K|=\frac{|N|}{\exp (N)}$.

Proof. Write $N=C \times H$ with $C$ cyclic of maximal order in $N$ and selected in such a way that if $p$ is prime and $\exp \left(N_{p}\right)=\exp \left(A_{p}\right)$, then $C_{p}=A_{p}$. We claim that if $K \in \mathbb{K}$, then $C \cap K=1$. Otherwise $C_{p} \cap K \neq 1$ for some prime $p$ and therefore $\exp \left(C_{p}\right)=\exp \left(N_{p}\right)>\exp \left(A_{p}\right)$. Let $x$ be a generator of $C_{p}, q=\left|A_{p}\right|$ and $a=(x, g)$ with $g \in G$. Then $a \in A_{p}$ and therefore $a^{q}=1$. Thus $\left(x^{q}\right)^{g}=x^{q}$. This proves that $x^{q}$ is a non-trivial central element of $G$. Then $Z(G) \cap K \neq 1$, contradicting the fact that $K$ does not contain any normal subgroup of $G$. This proves the claim.

Let $\pi_{1}$ and $\pi_{2}$ be the projections $N \rightarrow C$ and $N \rightarrow H$ along the decomposition $N=C \times H$. By the previous paragraph $K \cap \operatorname{ker} \pi_{2}=1$ and therefore $|K| \geq|H|=$ $\frac{|N|}{\exp (N)}$. As $N / K$ is cyclic we have $\exp (N) \leq[N: K]=\exp (N / K) \leq \exp (N)$. Hence $|K|=\frac{|N|}{\exp (N)}=|H|$ and therefore $\left.\pi_{2}\right|_{K}: K \rightarrow H$ is an isomorphism for every $K \in \mathbb{K}$. Therefore $K=\{f(h) h: h \in H\}$ for a homomorphism $f: H \rightarrow C$. (More precisely $f=\left.\pi_{1} \circ \pi_{2}\right|_{K} ^{-1}$.) Thus $K$ is completely determined by $f$ and hence $|\mathbb{K}| \leq|\operatorname{Hom}(H, C)|=|H|$. The last equality follows easily from the fact that $C$ is cyclic and $\exp (H)$ divides the order of $C$.

We are ready to prove our main result.

Theorem 3.4. If $G$ is a cyclic-by-abelian finite group then every normalized torsion unit of $\mathbb{Z} G$ is conjugate in $\mathbb{Q} G$ to an element of $G$.

Proof. By means of contradiction we assume that $G$ is a counterexample of minimal order of the theorem and $u$ is a normalized torsion unit of minimal order of $\mathbb{Z} G$ which is not conjugate to an element of $G$ in $\mathbb{Q} G$. We select a cyclic subgroup $A$ of $G$ with $G / A$ abelian and take $D=Z\left(C_{G}(A)\right)$ and $\mathbb{K}=\mathbb{K}_{D}$. By Proposition 1.1, we may assume without loss of generality that $\varepsilon_{x}(u)<0$ for some $x \in G$. This implies that the order of $x$ divides the order of $u$ by statement (2a) of Proposition 1.3. Set 
$m=|u|$ and $f=\left|\omega_{D}(u)\right|$. By assumption $u^{d}$ is conjugate in $\mathbb{Q} G$ to an element of $G$ for every $1 \neq d \mid m$.

By Lemma 2.3, $x \in D$ and by Corollary 2.6, $\omega_{D}(u) \neq 1$. Thus $1 \neq f \mid m$ and in particular, $u^{f}$ is conjugate in $\mathbb{Q} G$ to some $y \in D$. By the first induction hypothesis (ZC1) holds for every proper quotient of $G$ and hence we can use Lemma 3.1 for $N=D, u$ and $x$. Since $|K|=\frac{|D|}{\exp (D)}$ for every $K \in \mathbb{K}$, by Lemma 3.3, and $\left[\mathbb{Q}_{K}: \mathbb{Q}\right]=\varphi([D: K])$ we can write (3.8) for $h=f$ as

$$
\sum_{K \in \mathbb{K}} \mu_{\rho_{K}(u)}\left(\psi_{K}(x)\right)=\frac{\varphi(m)}{m} \frac{\left|C_{G}(x)\right|}{\varphi([D: K])} \varepsilon_{x}(u)+\frac{1}{f} \sum_{K \in \mathbb{K}} \mu_{\rho_{K}\left(u^{f}\right)}\left(\psi_{K}\left(x^{f}\right)\right) .
$$

We claim that

$$
\frac{1}{f} \sum_{k \in \mathbb{K}} \mu_{\rho_{K}\left(u^{f}\right)}\left(\psi_{K}\left(x^{f}\right)\right) \leq \frac{\varphi(m)}{m} \frac{\left|C_{G}(x)\right|}{\varphi([D: K])} .
$$

Write $f=f_{1} f_{2}$ with $f_{1}$ and $f_{2}$ positive integers such that the prime divisors of $f_{1}$ divide $|D|$ and $\left(f_{2},|D|\right)=1$. Then $m=f_{2} m^{\prime}$ with all prime divisors of $m^{\prime}$ dividing $|D|$. Note that $\left\langle x^{f}\right\rangle=\left\langle x^{f_{1}}\right\rangle$ and so $C_{G}\left(x^{f}\right)=C_{G}\left(x^{f_{1}}\right)$. Consider the map $\alpha: C_{G}\left(x^{f_{1}}\right) \rightarrow A$ given by $g \mapsto(x, g)$. If $a=(x, g)$ then $x^{g}=a x$ and therefore $x^{f_{1}}=\left(x^{f_{1}}\right)^{g}=a^{f_{1}} x^{f_{1}}$. Hence the image of $\alpha$ is contained in $\left\{a \in A: a^{f_{1}}=1\right\}$ and this is a subgroup of $A$ of order $\leq f_{1}$. On the other hand $\alpha(g)=\alpha(h)$ if and only if $g h^{-1} \in C_{G}(x)$. Therefore

$$
\left[C_{G}\left(x^{f_{1}}\right): C_{G}(x)\right] \leq f_{1} .
$$

Assume that $K \in \mathbb{K}$ and $y_{1}$ and $y_{2}$ are elements of $G$ in the same conjugacy class such that $y_{1} K=y_{2} K$. Then $y_{2} \in y_{1} A \cap y_{1} K=\left\{y_{1}\right\}$ because $A \cap K=1$. Therefore, if $C \in \mathcal{C}_{\mathbb{K}}$, then $\left\{g \in G:\left(x^{f}\right)^{g} \in y^{G} K_{C}\right\}$ is the disjoint union of the subsets $X_{C, y_{1}}=\left\{g \in G:\left(x^{f}\right)^{g} \in y_{1} K_{C}\right\}$ with $y_{1} \in y^{G}$. If $g, h \in X_{C, y_{1}}$ then $\left(x^{f}\right)^{g h^{-1}}=$ $\left(\left(x^{f}\right)^{h} k\right)^{h^{-1}}=x^{f} k^{h^{-1}}$ for some $k \in K_{C}$. Then $\left(x^{f}, g h^{-1}\right) \in A \cap K^{h^{-1}}=1$ and hence $g h^{-1} \in C_{G}\left(x^{f}\right)$. Conversely, if $g h^{-1} \in C_{G}\left(x^{f}\right)$ and $g \in X_{C, y_{1}}$ then $h \in X_{C, y_{1}}$. This proves that if $X_{C, y_{1}}$ is not empty, then it is a coset of $C_{G}\left(x^{f}\right)=C_{G}\left(x^{f_{1}}\right)$. Therefore for $u_{C}$ as in Lemma 3.1 we get

$$
\begin{aligned}
u_{C} & =\sum_{y_{1} \in y^{G}}\left|X_{C, y_{1}}\right| \leq\left|C_{G}\left(x^{f_{1}}\right)\right|\left|y^{G}\right|=\left|C_{G}\left(x^{f_{1}}\right)\right|\left[G: C_{G}(y)\right] \\
& \leq f_{1}\left|C_{G}(x)\right|\left[G: C_{G}(y)\right],
\end{aligned}
$$

and hence

$$
\sum_{C \in \mathcal{C}_{\mathbb{K}}} \frac{u_{C}}{\left|N_{G}\left(K_{C}\right)\right|} \leq f_{1} \frac{\left|C_{G}(x)\right|}{\left|C_{G}(y)\right|} \sum_{C \in \mathcal{C}_{\mathbb{K}}}\left[G: N_{G}\left(K_{C}\right)\right]=f_{1} \frac{\left|C_{G}(x)\right|}{\left|C_{G}(y)\right|}|\mathbb{K}| .
$$

Thus

$$
\frac{1}{f}\left[C_{G}(y): D\right] \sum_{C \in \mathcal{C}_{\mathbb{K}}} \frac{u_{C}}{\left|N_{G}\left(K_{C}\right)\right|} \leq \frac{1}{f_{2}}|\mathbb{K}|\left[C_{G}(x): D\right] .
$$

By Lemma $3.3|\mathbb{K}| \leq|K|$. Moreover, every prime divisor of $m^{\prime}$ divides $\exp (D)=$ $[D: K]$ and therefore

$$
\frac{1}{f_{2}} \leq \frac{\varphi\left(f_{2}\right)}{f_{2}} \frac{\varphi\left(m^{\prime}\right)}{m^{\prime}} \frac{[D: K]}{\varphi([D: K])}=\frac{\varphi(m)}{m} \frac{[D: K]}{\varphi([D: K])} .
$$


Thus

$$
\frac{1}{f_{2}}|\mathbb{K}|\left[C_{G}(x): D\right] \leq \frac{\varphi(m)}{m} \frac{[D: K]}{\varphi([D: K])}|K|\left[C_{G}(x): D\right]=\frac{\varphi(m)}{m} \frac{\left|C_{G}(x)\right|}{\varphi([D: K])}
$$

Combining (3.9), (3.17) and (3.19) we conclude that

$$
\begin{aligned}
\frac{1}{f} \sum_{k \in \mathbb{K}} \mu_{\rho_{K}\left(u^{f}\right)}\left(\psi_{K}\left(x^{f}\right)\right) & \leq \frac{1}{f}\left[C_{G}(y): D\right] \sum_{C \in \mathcal{C}_{K}} \frac{u_{C}}{\left|N_{G}\left(K_{C}\right)\right|} \\
& \leq \frac{\varphi(m)}{m} \frac{\left|C_{G}(x)\right|}{\varphi([D: K])} .
\end{aligned}
$$

This proves (3.13).

As the left side of (3.12) is non-negative we have

$$
\frac{1}{f} \sum_{k \in \mathbb{K}} \mu_{\rho_{K}\left(u^{f}\right)}\left(\psi_{K}\left(x^{f}\right)\right) \geq-\frac{\varphi(m)}{m} \frac{\left|C_{G}(x)\right|}{\varphi([D: K])} \varepsilon_{x}(u) \geq \frac{\varphi(m)}{m} \frac{\left|C_{G}(x)\right|}{\varphi([D: K])}
$$

because $\varepsilon_{x}(u)<0$. Therefore the equality holds in (3.13) and therefore the equality holds in all the inequalities from (3.14) to (3.20). This has the following consequences: $\varphi\left(f_{2}\right)=1$, so that $f_{2} \leq 2 ;\left|C_{G}\left(x^{f_{1}}\right)\right|=f_{1} C_{G}(x)$ and hence the conjugacy class of $x$ contains all the elements of the form $a^{i} x$, where $a$ is an element of $A$ of order $f_{1} ; m$ is divisible by all the primes dividing $|D| ; X_{C, y_{1}} \neq \emptyset$ for every $y_{1} \in y^{G}$; the left hand side of (3.12) is zero and hence $\psi_{K}(x)$ is not an eigenvalue of $\rho_{G}(u)$ for every $K \in \mathbb{K}$. Applying this to conjugates of $x$ we deduce that $\psi_{K}\left(x^{g}\right)$ is not an eigenvalue of $\rho_{K}(u)$ for every $g \in G$ and every $K \in \mathbb{K}$. We claim that $f_{2} \geq 0$ and all the eigenvalues of $\rho_{K}(u)$ have even order. If $\xi$ is an eigenvalue of $\rho_{K}(u)$ then $\xi^{f}$ is an eigenvalue of both $\rho_{K}\left(u^{f}\right)$ and $\rho_{K}(y)$. Thus $\xi^{f}=\psi_{K}\left(y_{1}\right)$ for some $y_{1} \in y^{G}$. As $X_{C, y_{1}} \neq \emptyset, \xi^{f}=\psi_{K}\left(\left(x^{f}\right)^{g}\right)$ for some $g \in G$ and therefore $\xi=\zeta_{f}^{j} \psi_{K}\left(x^{g}\right)$ for some $j$. However, $a^{i} x^{g}$ is conjugate to $x^{g}$ for every $0 \leq i<f_{1}$, where $a$ is an element of $A$ of order $f_{1}$. Hence $\psi_{K}\left(a^{i} x^{g}\right)=\zeta_{f_{1}}^{i} \psi_{K}\left(x^{g}\right)$ is not an eigenvalue of $\rho_{K}(u)$ for every $0 \leq i<f_{1}$. This implies that $f_{2}=2$ and $j$ is odd. Therefore the order of $\xi$ is even. This proves the claim. Then all the eigenvalues of $\rho_{K}\left(u^{\frac{m}{2}}\right)$ are equal to -1 and hence $\psi_{K}^{G}\left(u^{\frac{m}{2}}\right)=-[G: D]$. However $u^{\frac{m}{2}}$ is conjugate to an element of $G \backslash D$ and therefore $\psi_{K}^{G}\left(u^{\frac{m}{2}}\right)=0$, a contradiction.

\section{REFERENCES}

[BH08] V. Bovdi and M. Hertweck, Zassenhaus conjecture for central extensions of $S_{5}$, J. Group Theory 11 (2008), no. 1, 63-74. MR 2381018 (2009a:20010)

[BHK04] V. Bovdi, C. Höfert, and W. Kimmerle, On the first Zassenhaus conjecture for integral group rings, Publ. Math. Debrecen 65 (2004), no. 3-4, 291-303. MR 2107948 (2006f:20009)

[CR62] C.W. Curtis and I. Reiner, Representation theory of finite groups and associative algebras, Pure Appl. Math., Vol. XI, Interscience Pub., New York-London, 1962.

[CW00] G. Cliff and A. Weiss, Finite groups of matrices over group rings, Trans. Amer. Math. Soc. 352 (2000), no. 1, 457-475. MR 1608293 (2000c:20012)

[dRS06] Á. del Río and S.K. Sehgal, Zassenhaus conjecture (ZC1) on torsion units of integral group rings for some metabelian groups, Arch. Math. (Basel) 86 (2006), no. 5, 392397. MR 2229354 (2007c:16064)

[Her01] M. Hertweck, A counterexample to the isomorphism problem for integral group rings, Ann. of Math. 154 (2001), 115-138.

[Her06] On the torsion units of some integral group rings, Algebra Colloq. 13 (2006), no. 2, 329-348. MR 2208368 (2006k:16049) 
[Her08a] Torsion units in integral group rings of certain metabelian groups, Proc. Edinb. Math. Soc. (2) 51 (2008), no. 2, 363-385. MR 2465913 (2009j:16027)

[Her08b] Zassenhaus conjecture for $A_{6}$, Proc. Indian Acad. Sci. Math. Sci. 118 (2008), no. 2, 189-195. MR 2423231 (2009c:20010)

[HK06] C. Höfert and W. Kimmerle, On torsion units of integral group rings of groups of small order, Groups, rings and group rings, Lect. Notes Pure Appl. Math., vol. 248, Chapman \& Hall/CRC, Boca Raton, FL, 2006, pp. 243-252. MR 2226199 (2007d:16077)

[Kli91] L. Klingler, Construction of a counterexample to a conjecture of Zassenhaus, Comm. Algebra 19 (1991), no. 8, 2303-2330. MR 1123126 (92i:20004)

[LB83] I.S. Luthar and A.K. Bhandari, Torsion units of integral group rings of metacyclic groups, J. Number Theory 17 (1983), no. 2, 270-283. MR 716946 (85c:20004)

[LP89] I.S. Luthar and I.B.S. Passi, Zassenhaus conjecture for $A_{5}$, Proc. Indian Acad. Sci. Math. Sci. 99 (1989), no. 1, 1-5. MR 1004634 (90g:20007)

[LS98] I.S. Luthar and P. Sehgal, Torsion units in integral group rings of some metacyclic groups, Res. Bull. Panjab Univ. Sci. 48 (1998), no. 1-4, 137-153 (1999). MR 1773990 (2001f:16065)

[LT90] I.S. Luthar and P. Trama, Zassenhaus conjecture for certain integral group rings, J. Indian Math. Soc. (N.S.) 55 (1990), no. 1-4, 199-212. MR 1088139 (92b:20008)

[LT91] Z Zassenhaus conjecture for $S_{5}$, Comm. Algebra 19 (1991), no. 8, 2353-2362. MR 1123128 (92g:20003)

[MRSW87] Z. Marciniak, J. Ritter, S. K. Sehgal, and A. Weiss, Torsion units in integral group rings of some metabelian groups. II, J. Number Theory 25 (1987), no. 3, 340-352. MR 880467 (88k:20019)

[PMRS86] C. Polcino Milies, J. Ritter, and S.K. Sehgal, On a conjecture of Zassenhaus on torsion units in integral group rings. II, Proc. Amer. Math. Soc. 97 (1986), no. 2, 201-206. MR 835865 (87i:16013)

[PMS84] C. Polcino Milies and S.K. Sehgal, Torsion units in integral group rings of metacyclic groups, J. Number Theory 19 (1984), no. 1, 103-114. MR 751167 (86i:16009)

[Rob82] D.J.S. Robinson, A course in the theory of groups, Graduate Texts in Mathematics, vol. 80, Springer-Verlag, New York, 1982. MR 648604 (84k:20001)

[Rog91] K.W. Roggenkamp, Observations on a conjecture of Hans Zassenhaus, Groups-St. Andrews 1989, Vol. 2, London Math. Soc. Lecture Note Ser., vol. 160, Cambridge Univ. Press, Cambridge, 1991, pp. 427-444. MR 1123997 (92g:20004)

[RS83] J. Ritter and S.K. Sehgal, On a conjecture of Zassenhaus on torsion units in integral group rings, Math. Ann. 264 (1983), no. 2, 257-270. MR 711882 (85e:16014)

[Seh93] S.K. Sehgal, Units in integral group rings, Pitman Monographs and Surveys in Pure and Applied Mathematics, vol. 69, Longman Scientific \& Technical, Harlow, 1993, With an appendix by Al Weiss. MR 1242557 (94m:16039)

[Seh01] Z Zassenhaus conjecture, Encyclopaedia of mathematics. Supplement. Vol. III (M. Hazewinkel, ed.), Kluwer Academic Publishers, Dordrecht, 2001, pp. 453-454. MR 1935796 (2003j:00009)

[Seh03] Group rings, Handbook of algebra, Vol. 3, North-Holland, Amsterdam, 2003, pp. 455-541. MR 2035104 (2005d:16044)

[SW86] S.K. Sehgal and A. Weiss, Torsion units in integral group rings of some metabelian groups, J. Algebra 103 (1986), no. 2, 490-499. MR 864426 (88f:20015)

[Wei88] A. Weiss, Rigidity of p-adic p-torsion, Ann. of Math. (2) 127 (1988), no. 2, 317-332. MR 932300 (89g:20010)

[Wei91] _ Torsion units in integral group rings, J. Reine Angew. Math. 415 (1991), 175-187. MR 1096905 (92c:20009)

Departamento de Matemáticas, Universidad de Murcia, 30100 Murcia, Spain

E-mail address: mauriciojc02@hotmail.com

Fachbereich Mathematik, Universitaet Stuttgart, Pfaffenwaldring 57, 70569 Stuttgart, Germany

E-mail address: leo.imsueden@yahoo.com

Departamento de Matemáticas, Universidad de Murcia, 30100 Murcia, Spain

E-mail address: adelrio@um.es 